\title{
DEPREDACIÓN DE HEMIDACTYLUS MABOUIA (SQUAMATA: GEKKONIDAE) POR TROPIDOPHIS PARDALIS (SERPENTES: TROPIDOPHIIDAE)
}

\author{
Luis F. de Armas ${ }^{1}$ y Manuel Iturriaga ${ }^{2}$ \\ Instituto de Ecología y Sistemática, carretera de Varona No. 11835, entre Oriente y Lindero, \\ Reparto Parajón, Municipio Boyeros, La Habana 19, C.P. 11900, Cuba. \\ luisdearmas1945@gmail.com ${ }^{1}$, manueliturriaga@ecologia.cu²
}

\section{RESUMEN}

Se registra por primera vez la depredación, en vida libre, de la salamanquesa Hemidactylus mabouia (Moreau de Jonnés, 1818) por el Majacito Bobo Tropidophis pardalis (Gundlach, 1840). Además, se mencionan otros dos vertebrados, el lagarto Anolis porcatus Gray, 1841 (Squamata: Dactyloidae) y la ranita Eleutherodactylus planirostris (Cope, 1862), como presas de esta pequeña boa en la misma localidad.

Palabras clave: Anolis, Eleutherodactylus, boa enana, historia natural, Cuba, Antillas.

\section{PREDATION OF HEMIDACTYLUS MABOUIA (SQUAMATA: GEKKONIDAE) BY TROPIDOPHIS PARDALIS (SERPENTES: TROPIDOPHIIDAE)}

\section{ABSTRACT}

The predation of a free-living Tropical House Gecko Hemidactylus mabouia (Moreau de Jonnès, 1818) by the Spotted Brown Trope, Tropidophis pardalis (Gundlach, 1840), is recorded for the first time. Other two vertebrates, the Cuban Green Anole, Anolis porcatus Gray, 1841 (Squamata: Dactyloidae) and the small frog Eleutherodactylus planirostris (Cope, 1862) are also recorded as preys of this dwarf boa in the same locality.

Keywords: Anolis, Eleutherodactylus, dwarf boa, natural history, Cuba, West Indies.

Las pequeñas boas de la familia Tropidophiidae se distribuyen en Sudamérica y las Antillas (Pough et al., 2016); en Cuba están representadas por 16 especies del género Tropidophis Cocteau y Bibron, 1843, todas endémicas de este país antillano (Henderson y Powell, 2009).

El Majasito Bobo, Tropidophis pardalis (Gundlach, 1840), está ampliamente distribuido en toda Cuba, aunque hacia el extremo oriental es menos común (Rodríguez-Schettino et al., 2013). No obstante, como ocurre para la mayoría de sus congéneres cubanos, apenas existe información sobre los aspectos biológicos en condiciones naturales, estando limitadas casi todas las observaciones a las obtenidas en cautiverio (Polo y Moreno, 2010; Polo y Arango, 2011).

Hemidactylus mabouia (Moreau de Jonnés, 1818) es un gecko sinantrópico de origen africano, introducido accidentalmente en numerosos países tropicales, entre ellos Cuba (Weiss y Hedges, 2007; Henderson y Powell, 2009), donde se conoce mayormente como Salamanca o Salamanquesa (Silva, 1982; Buide, 1985), aunque en algunos lugares la llaman Lagartija de la Virgen. Es de hábitos nocturnos y vive mayormente asociado a las edificaciones humanas y sus alrededores, factores que probablemente condicionen la 
existencia de tan pocos depredadores conocidos [para Cuba no se ha registrado ninguno, aunque de Islas Vírgenes Británicas se han mencionado dos: el lagarto Anolis cristatellus Duméril y Bibron, 1837 y el ave paseriforme Margarops fuscatus (Vieillot, 1808); Owen y Perry, 2005; J. Lazell en Henderson y Powell, 2009].

El principal objetivo de la presente comunicación es dar a conocer un caso de depredación de $H$. mabouia por $T$. pardalis en un ambiente urbano, aunque también se mencionan como presas de esta serpiente a Anolis porcatus Gray, 1841 (Squamata: Dactyloidae) y Eleutherodactylus planirostris (Cope, 1862), Anura: Eleutherodactylidae, esta última registrada fotográficamente, ambas en la misma localidad del primer caso.

El 7 de marzo de 2017, a las 20:57 hr, el primer autor detectó en el patio de su casa, ubicada en San Antonio de los Baños $\left(22.89^{\circ} \mathrm{N}-82.50^{\circ} \mathrm{O} ; 70 \mathrm{msnm}\right)$, provincia de Artemisa, un adulto de $T$. pardalis que ingería un H. mabouia (Squamata: Gekkonidae); en esos momentos, ya había engullido la cabeza y casi todo el abdomen y ocho minutos más tarde ya casi había concluido de ingerirlo (fig. 1, A-B). Una vez concluida la ingestión del lagarto, a las 21:08 hr, se retiró lentamente, con cierta dificultad, posiblemente debido a la hartura. El ofidio medía aproximadamente $25 \mathrm{~cm}$ de longitud total. Algunas medidas del lagarto, tomadas in situ, fueron las siguientes (en milímetros): longitud y ancho basal de la cola $=74$ y 7.2, respectivamente; longitud del fémur de la pata posterior $=8.0$. Tanto la presa como su depredador fueron rápidamente atacados por hormigas Pheidole sp. (fig. 1, C-D); incluso, aun cuando el majasito se retiraba a su refugio, algunas permanecían prendidas a su cuerpo.

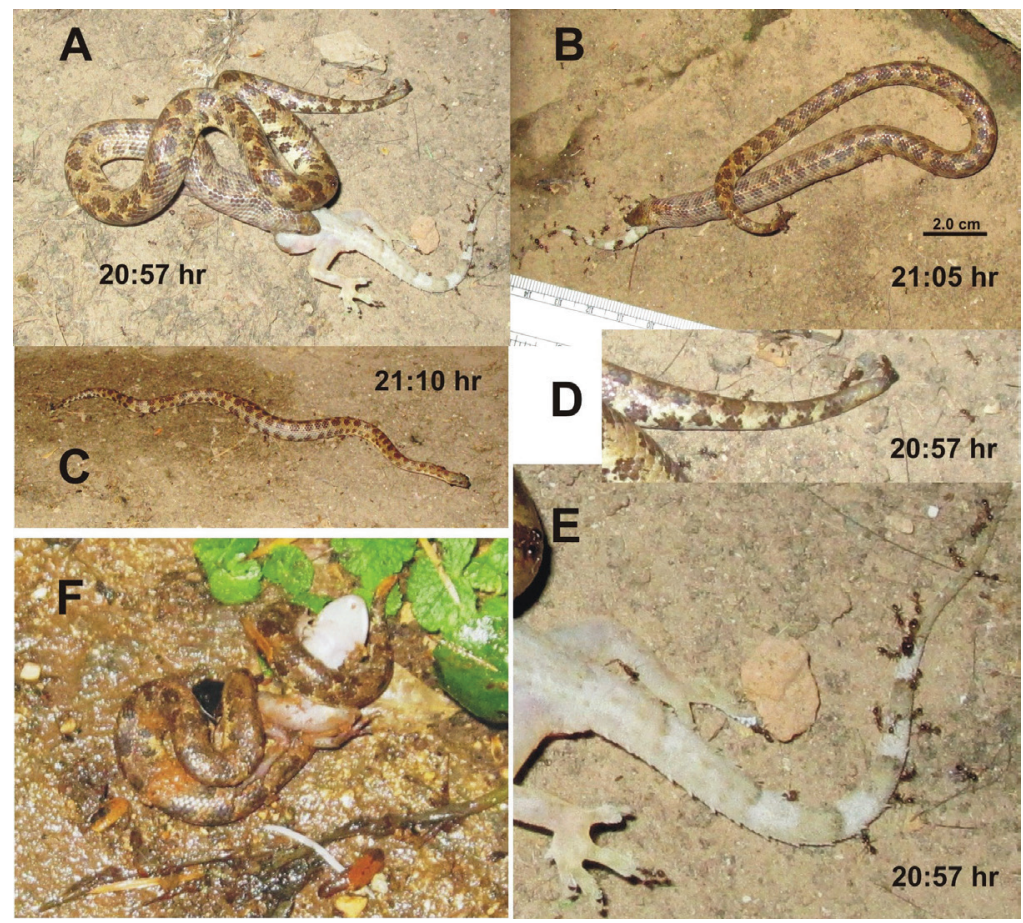

FIGURA 1. A-C, depredación de la salamanquesa Hemidactylus mabouia por el Majasito Bobo Tropidophis pardalis. A-B, fase final de la ingestión; C, concluida la ingestión de la salamanquesa, el majasito se retira a su refugio. D-E: detalles de la figura 1A, en los que se destaca el ataque de hormigas (Pheidole sp.) al majasito (D) y a su presa (E). F, Eleutherodactylus planirostris a punto de comenzar a ser engullido por T. pardalis. Ambos casos de depredación, en el patio de un domicilio urbano en San Antonio de los Baños, Artemisa, Cuba. 
En este mismo patio, el 5 de abril de 2013, a las 20:35 hr, un majasito adulto fue observado cuando terminaba de estrangular a una ranita (E. planirostris) y realizaba los primeros movimientos para localizar la cabeza de su presa y comenzar a engullirla (fig. 1E). Este representa el segundo depredador registrado para esta ranita en Cuba, además de la Rana Platanera, Osteopilus septendrionalis Duméril y Bibron, 1841 (Meshaka, 1996).

Otro caso observado en este sitio fue la depredación de un macho adulto de Anolis porcatus, hecho ocurrido a las 10:00 hr del 12 de mayo de 2010; una vez estrangulado el lagarto, comenzó a engullirlo por la cabeza, concluyendo de hacerlo totalmente 55 minutos más tarde. Schwartz y Henderson (1991) mencionaron que este majasito asecha a las lagartijas Anolis sp. durante la noche.

Estos dos últimos casos observados ocurrieron en el suelo, aunque estas pequeñas boas han sido halladas en el mismo patio hasta a $0.62 \mathrm{~m}$ de altura respecto al suelo, refugiadas debajo de las macetas ornamentales (LFA, observ. pers.). Con independencia de que las ranas y lagartos constituyen presas potenciales de T. pardalis, las aquí registradas representan las únicas hasta ahora confirmadas para la especie.

Aunque estas pequeñas boas son de hábitos eminentemente nocturnos (Schwartz y Henderson, 1991; Domínguez y Moreno, 2003), en reiteradas ocasiones han sido observadas al asecho durante las horas del día, casi siempre refugiadas debajo de piedras y con la cabeza asomada hacia el exterior, a la espera de una presa potencial (LFA, observ. pers.). La depredación del macho de $A$. porcatus aquí mencionada confirma que este majasito suele mantener su actividad de caza durante el horario diurno.

Una observación curiosa fue la realizada el día 24 de marzo de 2017, a las 20:32 hr, cuando un individuo de $T$. pardalis, de aproximadamente $20 \mathrm{~cm}$ de longitud total, fue visto mientras tomaba agua en el abrevadero de los animales domésticos del primer autor (fig. 2). Dicho bebedero está situado en el suelo, muy próximo a una pieza de hierro y consiste en una cazuela de aluminio de $21 \mathrm{~cm}$ de diámetro y $7.5 \mathrm{~cm}$ de profundidad; en esos momentos, el agua cubría solo $4.4 \mathrm{~cm}$ del fondo. El ofidio, que mantenía la parte posterior de su cuerpo sobre la pieza de hierro, descendió por el borde interno del abrevadero hasta acceder al líquido y estuvo bebiendo por más de cinco minutos. Aunque se trata de una única observación, el hecho sugiere que conductas similares pudieran desplegarse en la naturaleza, en los bordes de arroyos, lagunas y otros reservorios de agua, como los que se forman entre las rocas y depresiones del terreno después de la lluvia.

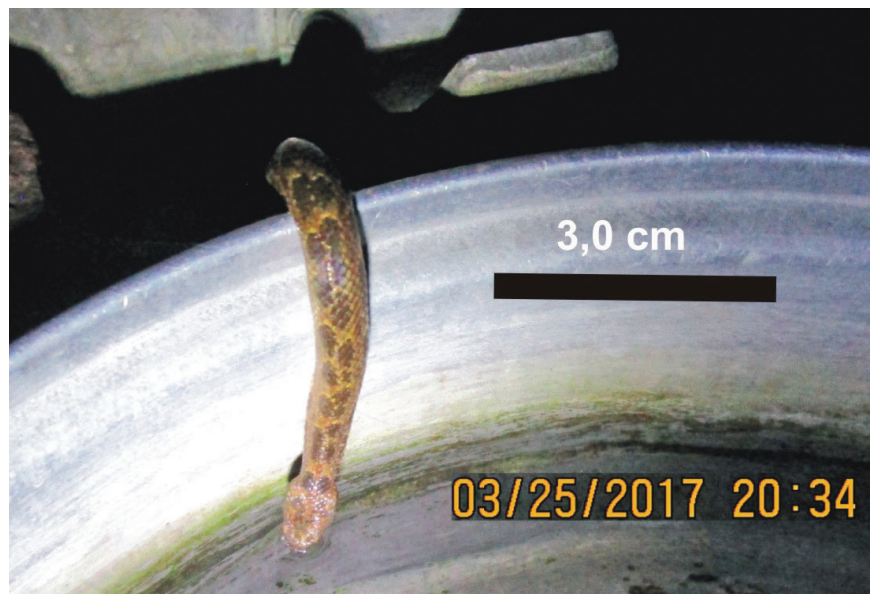

FIGURA 2. Tropidophis pardalis mientras bebe agua en el abrevadero de un perro, en el patio del primer autor (LFA). 
La presente contribución añade nueva información a la poca que hasta ahora existe sobre la historia natural de $T$. pardalis, a la vez que constituye el único caso registrado en Cuba de depredación de H. mabouia.

\section{LITERATURA CITADA}

Buide, M. 1985. Reptiles de Cuba. Editorial Gente Nueva, La Habana, 86 pp.

Domínguez Díaz, M. y L. V. Moreno. 2003. Serpientes del suelo. Pp. 98-109, en: Anfibios y reptiles de Cuba (L. Rodríguez Schettino, ed.). UPC Print, Vaasa, Finlandia, 169 pp.

Henderson, R. W. y R. Powell. 2009. Natural history of West Indian reptiles and amphibians. Gainesville, University of Florida Press, 495 pp.

Meshaka, W. E., Jr. 1996 Diet and the colonization of buildings by the Cuban Treefrog, Osteopilus septentrionalis (Anura: Hylidae). Caribbean Journal of Science, 32 (1): 59-63.

Owen, J. y G. Perry. 2005. Anolis cristatellus wileyae (Virgin Islands Crested Anole). Saurophagy. Herpetological Review, 36: 444.

Polo Leal, J. L. y A. Arango Leiva. 2011. Mantenimiento y reproducción del Majasito o Boa Leopardo Tropidophis pardalis, Gundlach, 1840 (Serpentes: Tropidophidae) en el Parque Zoológico Nacional de Cuba. Cubazoo, 24: 49-57.

Polo Leal, J. L. y L. V. Moreno. 2010. Serpientes de Cuba, ¿amigas o enemigas? Editorial Gente Nueva, La Habana, 91 pp.

Pough, F. H., R. M. Andrews, M. L. Crump, A. H. Savitzky, K. D. Wells y M. C. Brandley. 2016. Herpetology. Sinauer Associates, Inc, Sunderland, USA, 591 pp.

Rodríguez-Schettino, L., C. A. Mancina y V. G. Rivalta. 2013. Reptiles of Cuba: Checklist and Geographic Distributions. Smithsonian Herpetological Information Service, 144: 1-92.

Schwartz, A. y R. W. Henderson. 1991. Amphibians and reptiles of the West Indies. Descriptions, distributions and natural history. University of Florida Press, Gainesville, xvi +720 pp.

Silva Lee, A. 1982. Chipojos, bayoyas y camaleones. Editorial Gente Nueva, La Habana, 84 pp. + 64 láminas.

Weiss, A. J. y S. B. Hedges. 2007. Molecular phylogeny and biogeography of the Antillean geckos Phyllodactylus wishingi, Tarentola americana and Hemidactylus haitianus (Reptilia, Squamata). Molecular Phylogenetics and Evolution, 45: 409-416. 